\title{
Mode Grüneisen parameters of an efficient thermoelectric half-Heusler
}

Cite as: J. Appl. Phys. 124, 195107 (2018); https://doi.org/10.1063/1.5050697

Submitted: 02 August 2018 . Accepted: 02 November 2018 . Published Online: 20 November 2018

Hong-Jie Pang, Liu-Cheng Chen (D, Zi-Yu Cao, Hao Yu, Chen-Guang Fu, Tie-Jun Zhu (D), Alexander F. Goncharov (D), and Xiao-Jia Chen

\section{ARTICLES YOU MAY BE INTERESTED IN}

Multi-scale study of the deformation mechanisms of thermoelectric $p$-type half-Heusler

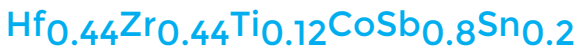

Journal of Applied Physics 124, 175104 (2018); https://doi.org/10.1063/1.5045591

Oxygen vacancy induced photoconductivity enhancement in $\mathrm{Bi}_{1-x} \mathrm{Ca}_{x} \mathrm{FeO}_{3-\delta}$ nanoparticle ceramics: A combined experimental and theoretical study

Journal of Applied Physics 124, 195108 (2018); https://doi.org/10.1063/1.5055742

High thermoelectric power factor of ytterbium silicon-germanium

Applied Physics Letters 113, 193901 (2018); https://doi.org/10.1063/1.5047091

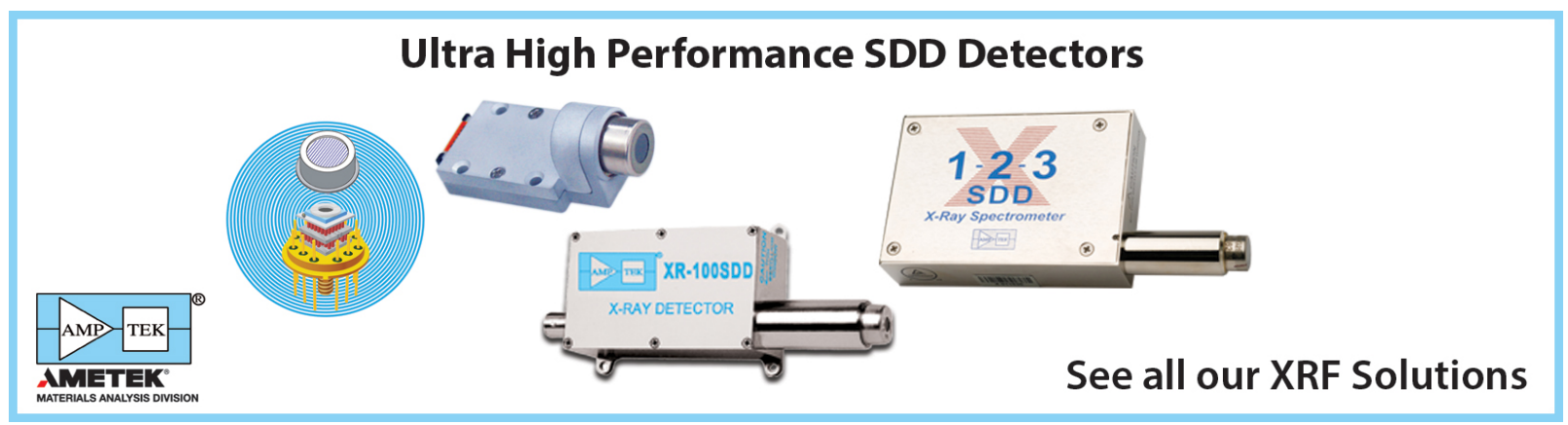




\title{
Mode Grüneisen parameters of an efficient thermoelectric half-Heusler
}

\author{
Hong-Jie Pang, ${ }^{1}$ Liu-Cheng Chen, ${ }^{1} \mathrm{Zi}-\mathrm{Yu}$ Cao, ${ }^{1} \mathrm{Hao} \mathrm{Yu},{ }^{1}$ Chen-Guang Fu, ${ }^{2,3}$ Tie-Jun Zhu, ${ }^{2}$ \\ Alexander F. Goncharov, ${ }^{4}$ and Xiao-Jia Chen ${ }^{1, a)}$ \\ ${ }^{1}$ Center for High Pressure Science and Technology Advanced Research, Shanghai 201203, China \\ ${ }^{2}$ State Key Laboratory of Silicon Materials, School of Materials Science and Engineering, Zhejiang University, \\ Hangzhou 310027, China \\ ${ }^{3}$ Max Planck Institute for Chemical Physics of Solids, 01187 Dresden, Germany \\ ${ }^{4}$ Geophysical Laboratory, Carnegie Institution of Washington, Washington, DC 20015, USA
}

(Received 2 August 2018; accepted 2 November 2018; published online 20 November 2018)

\begin{abstract}
Half-Heusler compounds have recently been identified as promising thermoelectric materials, but their relatively high thermal conductivities impede the further improvement of thermoelectric performance. The knowledge of phonon vibrational properties provides a fundamental understanding of the thermal transport behavior of solids and thus could serve as a guidance on further suppressing the thermal conductivity. Herein, a highly efficient $p$-type half-Heusler thermoelectric alloy $\mathrm{FeNb}_{0.8} \mathrm{Ti}_{0.2} \mathrm{Sb}$ is taken as an example to explore its phonon vibrational properties. Phonon spectrum with the frequencies down to $10 \mathrm{~cm}^{-1}$ and its evolution with pressure for the studied material are provided by Raman scattering. It is found that two vibrational modes with the frequency $>200 \mathrm{~cm}^{-1}$ display a common mode frequency increase with increasing pressure. Based on the bulk modulus from synchrotron X-ray powder diffraction and phonon frequency shifts, the mode Grüneisen parameters are obtained. Our results establish characteristic phonon vibrational properties of this high-performance half-Heusler thermoelectric alloy. Published by AIP Publishing. https://doi.org/10.1063/1.5050697
\end{abstract}

\section{INTRODUCTION}

Energy shortage is one of the most serious problems, and the increased burning of traditional fossil fuels will make air pollution more severe. Moreover, the waste heat is not effectively recovered. Thermoelectric materials can directly convert waste heat into useful electricity, providing a promising solution for energy conversion. The conversion efficiency of the thermoelectric materials is determined by the dimensionless figure of merit defined as ${ }^{1} z T=S^{2} \sigma T /\left(\kappa_{e}+\kappa_{l}\right)$, where $S, \sigma, \kappa_{e}, \kappa_{l}$, and $T$ are the Seebeck coefficient, electrical conductivity, electronic thermal conductivity, lattice thermal conductivity, and absolute temperature, respectively. The electrical and thermal transport parameters are interdependent $;^{2-5}$ therefore, it is a challenge to increase $z T$ by optimizing these parameters. Up to now, $z T=3$ is believed to be the ultimate goal for thermoelectrics to compete with traditional power generation. The half-Heuslers (HHs) with valance-electron-counts 8 and 18 are semiconductors and have been identified as great potential materials for moderate and high temperature thermoelectric applications ${ }^{6-15}$ due to the outstanding Seebeck coefficient and electrical conductivity. Additionally, the $\mathrm{HH}$ materials are promising for power generation due to the low cost, nontoxicity, and mechanical robustness. However, the relatively high thermal conductivity limits their thermoelectric performance. Recently, researchers found that alloying could reduce their thermal conductivities. ${ }^{16-22}$ Among them, $p$-type $\mathrm{HH}$ alloy $\mathrm{FeNb}_{0.8} \mathrm{Ti}_{0.2} \mathrm{Sb}$ is superior to the optimized typical $\mathrm{HH}$ compounds ${ }^{13,14,23}$ and its maximum

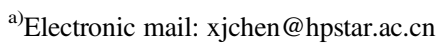

$z T(1.1$ at around $1100 \mathrm{~K})$ is almost twice as high as that of the most widely used $p$-type silicon-germanium thermoelectric materials. The achieved high power factor and a record output power density of $\sim 22 \mathrm{Wcm}^{-2}$ in such $p$-type $\mathrm{HH}$ compounds $^{14}$ are important for power generation applications. Investigation of the reasons behind the high thermal conductivity can provide a fundamental understanding of the thermal transport behavior of the material for future applications. Moreover, it can also provide clues for designing of new thermoelectric materials with low thermal conductivity.

Phonon spectrum provides the most direct information of the phonon vibrational properties ${ }^{24}$ and thus is useful for understanding and designing thermoelectric materials with low thermal conductivity. Experimental investigations of phonon properties are generally carried out by inelastic neutron scattering, inelastic X-ray scattering, and Raman scattering. The phonon information from Raman scattering has been proved to be identical to that from the other scattering techniques. ${ }^{25-27}$ As a powerful technique to study the phonon properties, Raman scattering has a high resolution and can be used to measure a small sample at high pressures. Therefore, it is desired to measure the phonon spectra by using this technique. It is known that strong lattice anharmonicity can significantly reduce the lattice thermal conductivity. ${ }^{28,27}$ Anomalous anharmonicity has also been established as the origin of low thermal conductivity. ${ }^{29}$ The lattice anharmonicity can be evaluated via measurements of the mode Grüneisen parameter. ${ }^{30}$ So far, experimental phonon spectra or Grüneisen parameters are not available for highly efficient thermoelectric $\mathrm{HH}$ materials. This is just the target of the current study. Here, we choose $\mathrm{FeNb}_{0.8} \mathrm{Ti}_{0.2} \mathrm{Sb}$ as an example to investigate the phonon vibrational properties. 


\section{EXPERIMENTAL DETAILS}

The sample preparation for $\mathrm{FeNb}_{0.8} \mathrm{Ti}_{0.2} \mathrm{Sb}$ was detailed elsewhere. ${ }^{13}$ The Raman spectra were collected with an excitation laser wavelength of $532 \mathrm{~nm}$ emitted by a Sapphire laser (Coherent) and analyzed by a Princeton Instrument spectrometer coupled to a Charge-Coupled-Device. The scattered light was focused on a $1800 \mathrm{~g} / \mathrm{mm}$ grating. The synchrotron X-ray powder diffraction studies were accomplished on PETRA III beamline P02.2 at DESY (Hamburg), a member of the Helmholtz Association using a wavelength of $0.2910 \AA$. The collected images were integrated by using Fit $2 \mathrm{D}^{31}$ in order to obtain the conventional diffraction patterns. The intensityversus- $2 \theta$ diffraction patterns were analyzed using a Rietveld refinement procedure employing GSAS software. ${ }^{32,33}$ The pressure was applied using a diamond anvil cell with neon as the pressure transmitting medium for both Raman scattering and $\mathrm{X}$-ray powder diffraction measurements. The pressure was measured by the ruby fluorescent method. ${ }^{34}$

\section{RESULTS AND DISCUSSION}

To obtain the Grüneisen parameter, we need to understand the compression behavior of the lattice parameters under pressure. Meanwhile, the data of pressure-induced structural evolution need to be acquired. Figure 1 shows the X-ray powder diffraction patterns at various pressures up to 42.4 GPa (upper panel) and the refined result of $\mathrm{FeNb}_{0.8} \mathrm{Ti}_{0.2} \mathrm{Sb}$ at a selected pressure (lower panel). With increasing pressure, all the diffraction peaks shift to higher angles indicating the

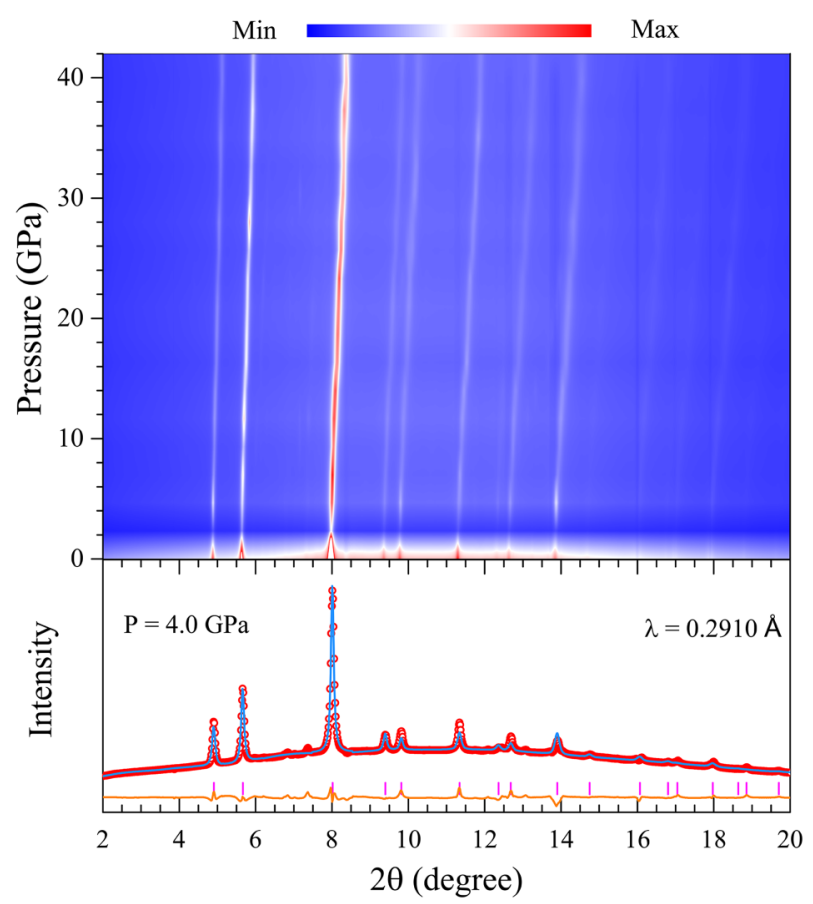

FIG. 1. Synchrotron X-ray $(\lambda=0.2910 \AA)$ powder diffraction patterns of $\mathrm{FeNb}_{0.8} \mathrm{Ti}_{0.2} \mathrm{Sb}$ at various pressures up to $42.4 \mathrm{GPa}$ (upper panel). The lower panel shows the refined X-ray powder diffraction pattern at $4.0 \mathrm{GPa}$. The open cycles represent the measured intensities, and the lines are the results of profile refinements. The positions of the Bragg reflections are marked by vertical lines, and the difference profiles are shown at the bottoms. The $R$ values are $R_{p}=0.04 \%, R_{w p}=0.08 \%$, and $\chi^{2}=1.15 \%$. shrinkage of the lattice. No new peaks appear or no peaks merge and/or split. There is no phase transition, indicating the stability of the sample at the pressure range studied. All the patterns can be well refined to the $F \overline{4} 3 m$ space group. As shown in Fig. 1 (lower panel), the lattice parameter, which is determined from the refinement at $4.0 \mathrm{GPa}$, is $a=5.892 \pm 0.001 \AA$ and the unit-cell volume $V$ is $204.597 \pm 0.106 \AA^{3}$. The atomic arrangement of the structure is presented in the inset of Fig. 2.

The obtained lattice parameter along the $a$ axis and the unit-cell volume $V$ of $\mathrm{FeNb}_{0.8} \mathrm{Ti}_{0.2} \mathrm{Sb}$ as a function of pressure are shown in Fig. 2. Error bars were marked at each point. The lattice evolution can be described by the Murnaghan equation of state through ${ }^{35} P=B_{0} \times\left[\left(V_{0} / V\right)^{B_{0}^{\prime}}-1\right] / B_{0}^{\prime}$, where $P$ is the pressure, $B_{0}$ is the bulk modulus, $B_{0}^{\prime}$ is its pressure derivative, and $V_{0}$ is the unit-cell volume at ambient pressure. This fitting yields $B_{0}=163.30 \pm 10.67$ GPa with $B_{0}{ }^{\prime}=5.19 \pm 0.78$ and $V_{0}=210.34 \pm 0.34 \AA^{3}$. These results are comparable to those obtained from theoretical calculations for undoped FeNbSb $\left(163.2 \mathrm{GPa}^{36}\right.$ and $\left.163 \mathrm{GPa}^{37}\right)$ and other HH compounds, such as $170 \mathrm{GPa}$ for RuNbSb. ${ }^{38}$ The robust stability of the lattice structure is important for maintaining the enhanced thermoelectric performance at high pressures. ${ }^{39,40}$

The Grüneisen parameter also requires the phonon spectrum and the evolution behavior of phonon frequency with pressure. This information is provided for the studied material by Raman scattering. Figure 3 presents the pressuredependent Raman spectra of $\mathrm{FeNb}_{0.8} \mathrm{Ti}_{0.2} \mathrm{Sb}$ from ambient pressure to high pressure $(42.7 \mathrm{GPa})$ at room temperature.

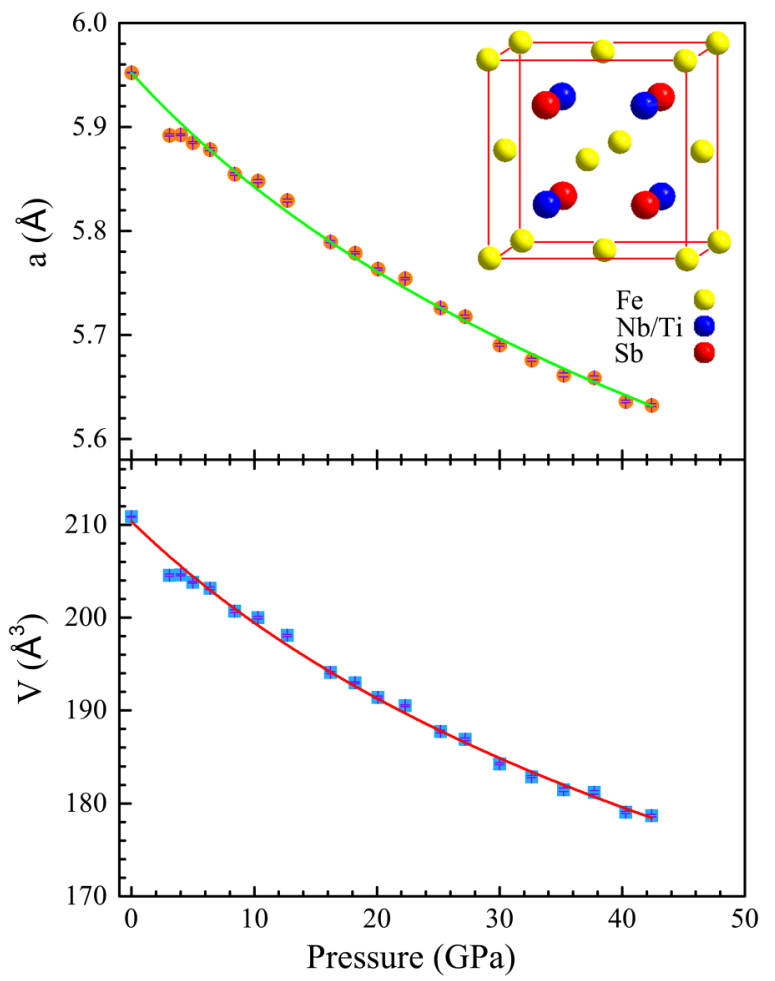

FIG. 2. The lattice parameter along the $a$ axis and the unit-cell volume $V$ of $\mathrm{FeNb}_{0.8} \mathrm{Ti}_{0.2} \mathrm{Sb}$ as a function of pressure. In all plots, error bars are smaller than the symbols. The solid symbols are the measured data points, and the curves are obtained from the fitting to the Murnaghan equation of state. Inset: Atomic arrangement of the $F \overline{4} 3 m$ structure. 


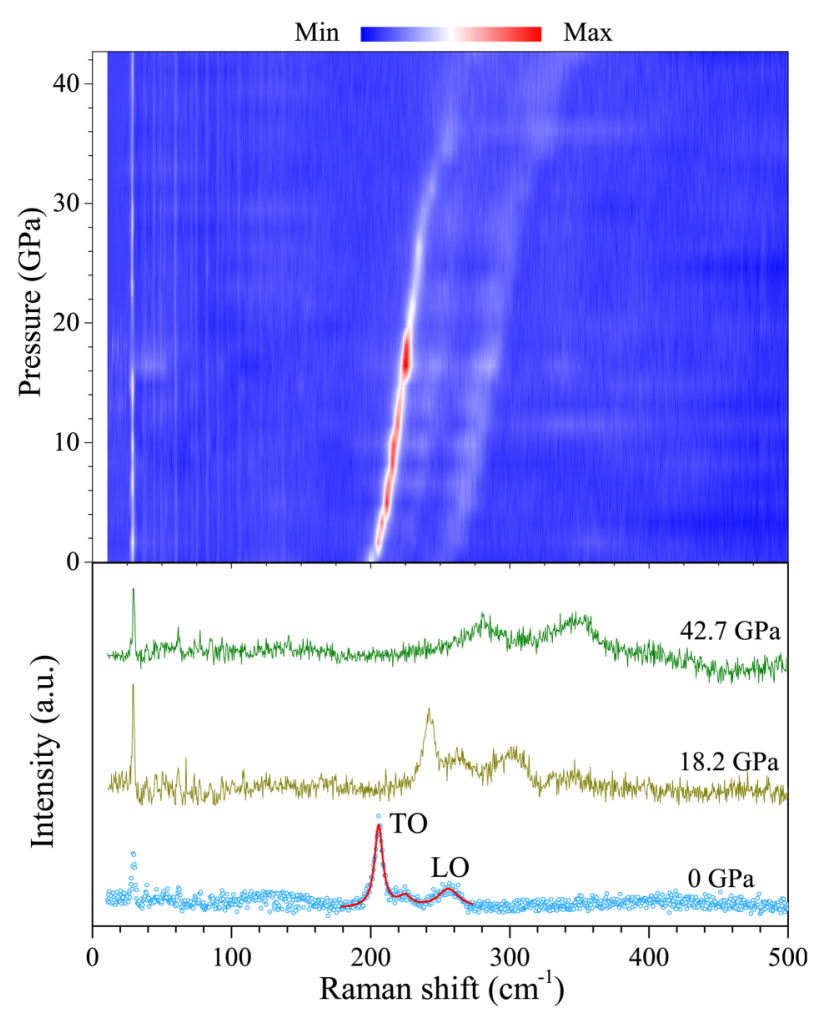

FIG. 3. Upper panel: Evolution of Raman spectra of $\mathrm{FeNb}_{0.8} \mathrm{Ti}_{0.2} \mathrm{Sb}$ with pressure up to $42.7 \mathrm{GPa}$ at room temperature. Lower panel: Selected Raman spectra at different pressures. The phonon frequencies of the TO and LO modes were obtained by Lorentz fitting.

The data were calculated by Lorentz fitting, as shown at the lower panel of Fig. 3. According to the group theory, three atoms in one primitive unit cell give rise to nine phonon branches, including one longitudinal acoustic (LA), two transverse acoustic (TA), two longitudinal optical (LO), and four transverse optical (TO) branches. In our experiment, one TO mode and one LO mode were observed. The assignments of the TO and LO modes are shown in Fig. 4. The arrows represent the vibrational directions of the atoms, yellow for $\mathrm{Fe}$, blue for $\mathrm{Nb} / \mathrm{Ti}$, and red for $\mathrm{Sb}$. The TO mode is the motions of $\mathrm{Nb} / \mathrm{Ti}$ against both counter atoms ( $\mathrm{Fe}$ and $\mathrm{Sb}$ ). The LO mode is a motion of Fe against $\mathrm{Sb}$. In comparison with the model assignments for $\mathrm{MeNiSn}^{41}$ it may be concluded that $\mathrm{Fe}$ and $\mathrm{Sb}$ symmetry coordinate contributions dominate the LO mode.

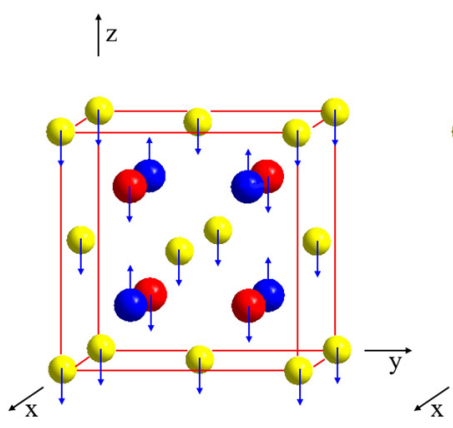

(a) TO mode

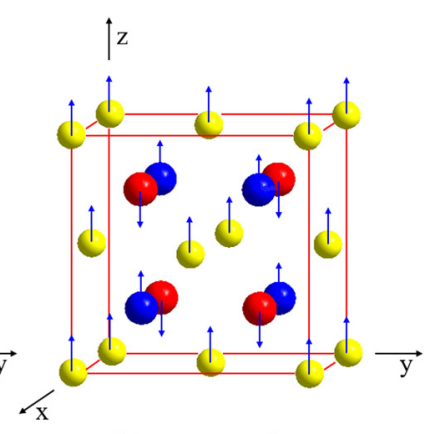

(b) LO mode
FIG. 4. Assignments for the TO and LO modes in a $\mathrm{FeNb}_{0.8} \mathrm{Ti}_{0.2} \mathrm{Sb}$ unit cell. The arrows represent the vibrational directions of the atoms, yellow for $\mathrm{Fe}$, blue for $\mathrm{Nb} / \mathrm{Ti}$, and red for $\mathrm{Sb}$.
A small peak with the frequency higher than the marked TO mode but with less intensity was found. This small peak is also the TO mode with different polarization. The appearance of the observed TO modes is probably due to the disorder. Due to the weak intensity, the assignment of the small peak is not shown. The general features of the obtained phonon spectra are consistent with the previous theoretical calculations of phonon dispersion ${ }^{37,42}$ and experiments. $^{41,43}$ The low frequency peak at about $30.0 \mathrm{~cm}^{-1}$ is unexpected. Unlike the high-frequency phonon modes, it does not depend on pressure. However, the position of this peak agrees well with the calculated acoustic phonon mode of the Brillouin zone boundary acoustic branches. ${ }^{37}$ Due to a disorder, the Raman selection rules are relaxed, which results in observation of an additional Raman band in the position corresponding to the maximum in density of the phonon states. This indicates the nano-crystalline feature of the synthesized samples.

As shown in Fig. 3, the intensities of all the phonon modes decrease with increasing pressure. The Raman spectra show no evidence of phase transition, which is consistent with the X-ray powder diffraction results. Meanwhile, all the phonon modes shift to higher frequencies. An energy gap between the bands which are originated from acoustic and optical phonon branches is observed for this HH compound. The energy gap increases with increasing pressure. The result emphasizes the possibility to tune the phonon dispersion just through lattice compression. This is of interest when investigating strategies to obtain a low lattice thermal conductivity.

Previous theoretical calculations on lattice dynamics also call for the experiments for the comparison. ${ }^{24,37,38,42,44}$ Hereby, we give a direct measure of the Grüneisen parameters for the phonon modes from the obtained Raman spectra. The Grüneisen parameter describes the volume dependence of phonon frequency $\omega_{i}$, defined as $\gamma_{i}=-d \ln \omega_{i} /$ $d \ln V=\left(B_{0} / \omega_{i}\right) \times\left(d \omega_{i} / d P\right)$. Because Grüneisen parameter is a measure of the departure of a crystal from harmonicity, different vibrational modes will have different values of Grüneisen parameter.

Figure 5 illustrates the pressure dependence of the frequencies of $\mathrm{FeNb}_{0.8} \mathrm{Ti}_{0.2} \mathrm{Sb}$ for $\mathrm{TO}$ and $\mathrm{LO}$ modes at room temperature. Because the structure remains stable, it is clear that the pressure-induced frequency shift is mainly due to the phonon anharmonicity. ${ }^{30}$ In order to obtain the mode-Grüneisen parameter for each phonon mode, we fitted the pressure dependence of phonon frequencies in Fig. 5 in a quadratic formula: $\omega_{i}(P)=\omega_{i 0}+\left(d \omega_{i} / d P\right) P-\left(d^{2} \omega_{i} / d P^{2}\right) P^{2}$.

As a result, the more practical Grüneisen parameter of each phonon mode could be obtained from experimental data. The obtained phonon frequencies, their pressure derivatives, and Grüneisen parameters for each phonon mode are listed in Table I. As can be seen, the values of the mode Grüneisen parameters are very close for all the modes. The positive $\gamma_{i}$ values for these modes are the signatures for the optical modes. These results are also consistent with the theoretical calculations for the sister $\mathrm{HH}$ compound, ${ }^{24}$ altogether implying that our assignment of the phonon modes for the studied compound is reasonable. 


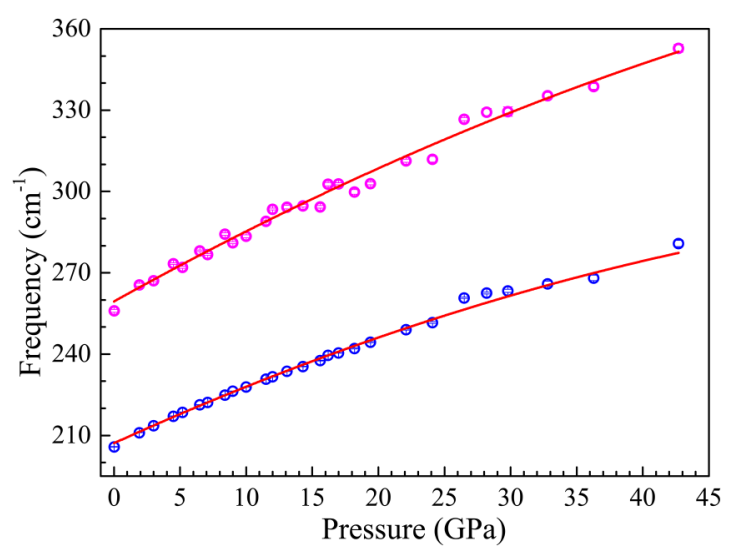

FIG. 5. Pressure dependence of the frequencies of the phonon modes of $\mathrm{FeNb}_{0.8} \mathrm{Ti}_{0.2} \mathrm{Sb}$. The solid lines are the fitted curves to the experimental data points.

A large Grüneisen parameter means strong anharmonicity, which can give rise to low lattice thermal conductivity in ordered crystal structures. We further calculate the average Grüneisen parameter of the two phonon modes based on $\bar{\gamma}=\sqrt{\left(\gamma_{i}^{2}\right)}$. The value of the $\bar{\gamma}$ for the studied modes is about $1.72 \pm 0.43$. Interestingly, the obtained $\bar{\gamma}$ is close to the calculated value of 1.71 for a HH compound ScRhTe. ${ }^{44}$ To our knowledge, this is the first experimental data so far available for $\mathrm{HH}$ materials, ${ }^{24,37,38,42,44}$ especially for highly efficient $\mathrm{HH}$ alloys. It should provide a direct measure of the quantitative understanding of the thermal conductivity behavior as well as the thermoelectric properties in this $\mathrm{HH}$ family.

Due to the difficulties of measurements, the available Grüneisen parameters for the thermoelectric materials in the literature are usually obtained from theoretical calculations $^{24,37,38,42,44}$ and the combination of different experimental techniques. ${ }^{4,45}$ The bulk thermoelectric materials often include defects or disorders or some nano domains. Theoretical treatments become difficult if all these factors would be included in the accurate determination of the real phonon spectra of a material. The obtained phonon spectra and Grüneisen parameters for $\mathrm{FeNb}_{0.8} \mathrm{Ti}_{0.2} \mathrm{Sb}$ are based on the same sample and measurement environment (pressure medium). The determined mode Grüneisen parameters should capture the basic character of the phonon frequency reaction to the lattice change. The value of $\bar{\gamma}$ for $\mathrm{FeNb}_{0.8} \mathrm{Ti}_{0.2} \mathrm{Sb}$ is smaller than the well-known low lattice thermal conductivity materials $\mathrm{AgSbTe}_{2},{ }^{46} \mathrm{AgSbSe}_{2},{ }^{46}$ and $\mathrm{SnSe}$ (theoretical value of 4.1 along the $a$ axis). ${ }^{28}$ It reveals that anharmonicity contributes to the relatively high thermal conductivity of $\mathrm{HH}$ materials compared to other thermoelectric materials. ${ }^{47}$ However, the value of $\bar{\gamma}$ is higher than

TABLE I. Summary of the vibrational modes, frequency $\omega_{i 0}\left(\mathrm{~cm}^{-1}\right)$, pressure derivative $d \omega_{i} / d P\left(\mathrm{~cm}^{-1} / \mathrm{GPa}\right), d^{2} \omega_{i} / d P^{2}\left(\times 10^{-2} \mathrm{~cm}^{-1} / \mathrm{GPa}^{2}\right)$, and mode-Grüneisen parameter $\gamma_{i}$ for each phonon mode of $\mathrm{FeNb}_{0.8} \mathrm{Ti}_{0.2} \mathrm{Sb}$.

\begin{tabular}{lcccc}
\hline \hline Mode & $\omega_{i 0}$ & $d \omega_{i} / d P$ & $d^{2} \omega_{i} / d P^{2}$ & $\gamma_{i}$ \\
\hline TO & $207.1 \pm 0.3$ & $2.2 \pm 0.1$ & $0.01 \pm 0.0$ & $1.73 \pm 0.19$ \\
LO & $259.4 \pm 1.2$ & $2.7 \pm 0.2$ & $0.01 \pm 0.0$ & $1.70 \pm 0.23$ \\
\hline \hline
\end{tabular}

$\mathrm{PbTe}^{45}$ and $\mathrm{Bi}_{2} \mathrm{Te}_{3}{ }^{48}$ which are conventional low lattice thermal conductivity materials. This implies that anharmonicity alone is not sufficient to account for the relatively high thermal conductivity of $\mathrm{HH}$ materials.

In a recent experimental study, ${ }^{23}$ we found that electrons contribute more than lattice to the thermal conductivity above $200 \mathrm{~K}$. It is obvious that the thermal conductivity is governed by electronic transports at high temperatures. Meanwhile, the disorder was found to affect the electrical and thermal transport properties in the system. ${ }^{23}$ Therefore, the thermal conductivity is more likely controlled by other factors as well.

\section{CONCLUSIONS}

In summary, we have performed X-ray powder diffraction and high-resolution Raman scattering measurements on $\mathrm{FeNb}_{0.8} \mathrm{Ti}_{0.2} \mathrm{Sb}$ at various pressures up to $42 \mathrm{GPa}$. The $F \overline{4} 3 m$ phase is found to be stable at the pressure range studied. The bulk modulus $\left(B_{0}=163.30 \pm 10.67 \mathrm{GPa}\right)$ of $\mathrm{FeNb}_{0.8} \mathrm{Ti}_{0.2} \mathrm{Sb}$ at ambient pressure and $300 \mathrm{~K}$ is determined through the equation of state. Raman spectra and their evolution with pressure were measured from ambient pressure to $42.7 \mathrm{GPa}$. Based on the bulk modulus and the phonon frequency shifts, the Grüneisen parameters for each observed phonon mode at $300 \mathrm{~K}$ are further obtained. The results are used to understand the obtained thermal conductivity in the studied compound. These findings are crucial for a fundamental understanding of thermal transport in one of the best performance thermoelectric materials.

\section{ACKNOWLEDGMENTS}

The sample preparation was supported by the National Natural Science Foundation of China (No. 11574267). The synchrotron X-ray powder diffraction measurements were performed on PETRA III beamline P02.2 at DESY (Hamburg), a member of the Helmholtz Association (HGF). The authors are grateful to Anna Pakhomova for technical assistance in experiments.

${ }^{1}$ D. S. Lieberman, "Thermoelectricity," Phys. Today 12(2), 26 (1959).

${ }^{2}$ G. J. Snyder and E. S. Toberer, "Complex thermoelectric materials," Nat. Mater. 7, 105 (2008).

${ }^{3}$ X. Shi, L. D. Chen, and C. Uher, "Recent advances in high-performance bulk thermoelectric materials," Int. Mater. Rev. 61, 379 (2016).

${ }^{4}$ Z. F. Ren, Y. C. Lan, and Q. Y. Zhang, Advanced Thermoelectrics: Materials, Contacts, Devices, and Systems (CRC Press, 2017).

${ }^{5}$ T. J. Zhu, Y. T. Liu, C. G. Fu, J. P. Heremans, J. G. Snyder, and X. B. Zhao, "Compromise and synergy in high-efficiency thermoelectric materials," Adv. Mater. 29, 1605884 (2017).

${ }^{6} \mathrm{~S}$. Chen and Z. F. Ren, "Recent progress of half-Heusler for moderate temperature thermoelectric applications," Mater. Today 16, 387 (2013).

${ }^{7}$ D. P. Young, P. Khalifah, and R. J. Cava, "Thermoelectric properties of pure and doped FeMSb (M=V,Nb)," J. Appl. Phys. 87, 317 (2000).

${ }^{8}$ J. Yang, H. Li, T. Wu, W. Q. Zhang, L. D. Chen, and J. H. Yang, "Evaluation of half-Heusler Compounds as thermoelectric materials based on the calculated electrical transport properties," Adv. Funct. Mater. 18, 2880 (2008).

${ }^{9}$ S. Populoh, M. H. Aguirre, O. C. Brunko, K. Galazka, Y. Lu, and A. Weidenkaff, "High figure of merit in (Ti, Zr, Hf) half-Heusler alloys," Scr. Mater. 66, 1073 (2012).

${ }^{10}$ S. Chen, K. C. Lukas, W. S. Liu, C. P. Opeil, G. Chen, and Z. F. Ren, "Effect of Hf Concentration on thermoelectric properties of nanostructured 
n-type half-Heusler materials $\mathrm{Hf}_{x} \mathrm{Zr}_{1-x} \mathrm{NiSn}_{0.99} \mathrm{Sb}_{0.01}, " \mathrm{Adv}$. Energy Mater. 3, 1210 (2013).

${ }^{11}$ T. J. Zhu, C. G. Fu, H. H. Xie, Y. T. Liu, and X. B. Zhao, "High efficiency half-Heusler thermoelectric materials for energy harvesting," Adv. Energy Mater. 5, 1500588 (2015).

${ }^{12}$ C. G. Fu, S. Q. Bai, Y. T. Liu, Y. S. Tang, L. D. Chen, X. B. Zhao, and T. J. Zhu, "Realizing high figure of merit in heavy-band $p$-type half-Heusler thermoelectric materials," Nat. Commun. 6, 8144 (2015).

${ }^{13}$ C. G. Fu, T. J. Zhu, Y. T. Liu, H. H. Xie, and X. B. Zhao, "Band engineering of high performance $p$-type FeNbSb based half-Heusler thermoelectric materials for figure of merit zT > 1," Energy Environ. Sci. 8, 216 (2015).

${ }^{14}$ R. He, D. Kraemer, J. Mao, L. P. Zeng, Q. Jie, Y. C. Lan, C. H. Li, J. Shuai, H. S. Kim, Y. Liu, D. Broido, C. W. Chu, G. Chen, and Z. F. Ren, "Achieving high power factor and output power density in p-type half-Heuslers $\mathrm{Nb}_{1-x} \mathrm{Ti}_{x} \mathrm{FeSb}$," Proc. Natl. Acad. Sci. U.S.A. 113, 13576 (2016).

${ }^{15}$ R. He, H. T. Zhu, J. Y. Sun, J. Mao, H. Reith, S. Chen, G. Schiernin, K. Nielsch, and Z. F. Ren, "Improved thermoelectric performance of $n$-type half-Heusler $\mathrm{MCo}_{1-x} \mathrm{Ni}_{x} \mathrm{Sb}(\mathrm{M}=\mathrm{Hf}, \mathrm{Zr})$," Mater. Today Phys. 1, 24 (2017).

${ }^{16}$ C. Uher, J. Yang, S. Hu, D. T. Morelli, and G. P. Meisner, "Transport properties of pure and doped MNiSn $(\mathrm{M}=\mathrm{Zr}$, Hf)," Phys. Rev. B 59, 8615 (1999).

${ }^{17}$ L. Jodin, J. Tobola, P. Pecheur, and H. Scherrer, "Effect of substitutions and defects in half-Heusler FeVSb studied by electron transport measurements and KKR-CPA electronic structure calculations," Phys. Rev. B 70, 184207 (2004).

${ }^{18}$ T. J. Zhu, K. Xiao, C. Yu, J. J. Shen, S. H. Yang, A. J. Zhou, and J. He, "Effects of yttrium doping on the thermoelectric properties of half-Heusler alloys $\mathrm{Hf}_{0.6} \mathrm{Zr}_{0.4} \mathrm{NiSn}_{0.98} \mathrm{Sb}_{0.02}$, J. Appl. Phys. 108, 044903 (2010).

${ }^{19}$ C. G. Fu, H. H. Xie, T. J. Zhu, J. Xie, and X. B. Zhao, "Enhanced phonon scattering by mass and strain field fluctuations in $\mathrm{Nb}$ substituted $\mathrm{FeVSb}$ half-Heusler thermoelectric materials," J. Appl. Phys. 112, 124915 (2012).

${ }^{20}$ H. H. Xie, H. Wang, Y. Z. Pei, C. G. Fu, X. H. Liu, G. J. Synder, X. B. Zhao, and T. J. Zhu, "Beneficial contribution of alloy disorder to electron and phonon transport in half-Heusler thermoelectric materials," Adv. Funct. Mater. 23, 5123 (2013).

${ }^{21}$ C. G. Fu, Y. T. Liu, H. H. Xie, X. H. Liu, X. B. Zhao, G. J. Snyder, J. Xie, and T. J. Zhu, "Electron and phonon transport in Co-doped $\mathrm{FeV}_{0.6} \mathrm{Nb}_{0.4} \mathrm{Sb}$ half-Heusler thermoelectric materials," J. Appl. Phys. 114, 134905 (2013).

${ }^{22}$ C. G. Fu, H. J. Wu, Y. T. Liu, J. Q. He, X. B. Zhao, and T. J. Zhu, "Enhancing the figure of merit of heavy-band thermoelectric materials through hierarchical phonon scattering," Adv. Sci. 3, 1600035 (2016).

${ }^{23}$ H. J. Pang, C. G. Fu, H. Yu, L. C. Chen, T. J. Zhu, and X. J. Chen, "Origin of efficient thermoelectric performance in half-Heusler $\mathrm{FeNb}_{0.8} \mathrm{Ti}_{0.2} \mathrm{Sb}$," J. Appl. Phys. 123, 235106 (2018).

${ }^{24}$ J. Shiomi, K. Esfarjani, and G. Chen, "Thermal conductivity of half-Heusler compounds from first-principles calculations," Phys. Rev. B 84, 104302 (2011)

${ }^{25}$ X. J. Chen, V. V. Struzhkin, S. Kung, H. K. Mao, R. J. Hemley, and A. N. Christensen, "Pressure-induced phonon frequency shifts in transitionmetal nitrides," Phys. Rev. B 70, 014501 (2004).

${ }^{26}$ M. Christensen, A. B. Abrahamsen, N. B. Christensen, F. Juranyi, N. H. Andersen, K. Lefmann, J. Andreasson, C. R. H. Bahl, and B. B. Iversen, "Avoided crossing of rattler modes in thermoelectric materials," Nat. Mater. 7, 811 (2008).

${ }^{27}$ C. W. Li, J. Hong, A. F. May, D. Bansal, S. Chi, T. Hong, G. Ehlers, and O. Delaire, "Orbitally driven giant phonon anharmonicity in SnSe," Nat. Phys. 11, 1063 (2015).
${ }^{28}$ L. D. Zhao, S. H. Lo, Y. S. Zhang, H. Sun, G. J. Tan, C. Uher, C. Wolverton, V. P. Dravid, and M. G. Kanatzidis, "Ultralow thermal conductivity and high thermoelectric figure of merit in SnSe crystals," Nature 508, 373 (2014).

${ }^{29}$ Y. Tian, S. Jia, R. J. Cava, R. D. Zhong, J. Schneeloch, G. Gu, and K. S. Burch, "Understanding the evolution of anomalous anharmonicity in $\mathrm{Bi}_{2} \mathrm{Te}_{3-x} \mathrm{Se}_{x} \mathrm{O}$," Phys. Rev. B 95, 094104 (2017).

${ }^{30}$ W. F. Sherman, "Bond anharmonicities, Grüneisen parameters, pressureinduced frequency shifts," J. Phys. C: Solid State Phys. 13, 4601 (1980).

${ }^{31}$ A. P. Hammersley, S. O. Svensson, M. Hanfland, A. N. Fitch, and D. Hausermann, "Two-dimensional detector software: From real detector to idealised image or two-theta scan," High Press Res. 14, 235 (1996).

${ }^{32}$ A. C. Larson and R. B. Von Dreele, GSAS (general structure analysis system), Los Alamos National Laboratory Report LAUR 86-748, 1994, see http://netlib.ccp14.ac.uk/ccp/web-mirrors/pssp/pdf/GSASManual.pdf.

${ }^{33}$ B. H. Toby, "EXPGUI, a graphical user interface for GSAS," J. Appl. Cryst. 34, 210 (2001).

${ }^{34}$ H. K. Mao, P. M. Bell, J. W. Shaner, and D. J. Stembey, "Specific volume measurements of $\mathrm{Cu}, \mathrm{Mo}, \mathrm{Pd}$, and $\mathrm{Ag}$ and calibration of the ruby R1 fluorescence pressure gauge from 0.06 to 1 Mbar," J. Appl. Phys. 49, 3276 (1978).

${ }^{35}$ F. D. Murnaghan, "The compressibility of media under extreme pressure," Proc. Natl. Acad. Sci. U.S.A. 30, 244 (1944).

${ }^{36}$ G. Joshi, R. He, M. Engber, G. Samsonidze, T. Pantha, E. Dahal, K. Dahal, J. Yang, Y. C. Lan, B. Kozinsky, and Z. F. Ren, "NbFeSb-based $p$-type half-Heuslers for power generation applications," Energy Environ. Sci. 7, 4070 (2014)

${ }^{37}$ C. Çoban, K. Çolakoğlu, and Y. Ö. Çiftçi, "First principles study of the structural, mechanical, phonon, optical, and thermodynamic properties of half-Heusler (HH) compound NbFeSb," Phys. Scr. 90, 095701 (2015).

${ }^{38}$ T. Fang, S. Q. Zheng, T. Zhou, L. Yan, and P. Zhang, "Computational prediction of high thermoelectric performance in $p$-type half-Heusler compounds with low band effective mass," Phys. Chem. Chem. Phys. 19, 4411 (2017).

${ }^{39}$ H. Yu, L. C. Chen, H. J. Pang, X. Y. Qin, P. F. Qiu, X. Shi, L. D. Chen, and X. J. Chen, "Large enhancement of thermoelectric performance in CuInTe 2 upon compression," Mater. Today Phys. 5, 1 (2018).

${ }^{40}$ L. C. Chen, H. Yu, H. J. Pang, B. B. Jiang, L. Su, X. Shi, L. D. Chen, and X. J. Chen, "Pressure-induced enhancement of thermoelectric performance in palladium sulfide," Mater. Today Phys. 5, 64 (2018).

${ }^{41}$ Z. V. Popović, G. Kliche, R. Liu, and F. G. Aliev, "Vibrational properties of MeNiSn (Me = Ti,Zr,Hf)," Solid State Commun. 74, 829 (1990).

${ }^{42}$ A. J. Hong, L. Li, R. He, J. J. Gong, Z. B. Yan, K. F. Wang, J. M. Liu, and Z. F. Ren, "Full-scale computation for all the thermoelectric property parameters of half-Heusler compounds," Sci. Rep. 6, 22778 (2016).

${ }^{43}$ N. Mestres, J. M. Calleja, F. G. Aliev, and A. I. Belogorokhov, "Electron localization in the disordered conductors TiNiSn and HfNiSn observed by Raman and infrared spectroscopies," Solid State Commun. 91, 779 (1994).

${ }^{44}$ S. Singh, "Assessing the thermoelectric properties of ScRhTe half-heusler compound," Comput. Condens. Matter 13, 120 (2017).

${ }^{45}$ G. A. Slack, in Solid State Physics: Advances in Research and Applications, edited by H. Ehrenreich, F. Seitz, and D. Turnbull (Academic Press, New York, 1979).

${ }^{46}$ D. T. Morelli, V. Jovovic, and J. P. Heremans, "Intrinsically minimal thermal conductivity in cubic I-V-VI 2 semiconductors," Phys. Rev. Lett. 101, 035901 (2008).

${ }^{47}$ L. C. Chen, Z. Y. Cao, H. Yu, B. B. Jiang, L. Su, X. Shi, L. D. Chen, and $\mathrm{X}$. J. Chen, "Phonon anharmonicity in thermoelectric palladium sulfide by Raman spectroscopy," Appl. Phys. Lett. 113, 022105 (2018).

${ }^{48}$ J. O. Jenkins, J. A. Rayne, and R. W. Ure, "Elastic moduli and phonon properties of $\mathrm{Bi}_{2} \mathrm{Te}_{3}$, , Phys. Rev. B 5, 3171 (1972). 